\title{
Diversity Consciousness: Opening Our Minds To People, Cultures, And Opportunities
}

By Richard D. Bucher

Upper Saddle River, NJ, 2000, 233 pages

Reviewed by

\section{Myron L. Pope}

Diversity Consciousness is a valuable public policy report addressed to professionals in higher education regarding a very significant topic: diversity. These professionals at institutions of higher and modernistic thinking must be able to identify, analyze and react to the trends which their environment presents. One of the more current issues and realities is that of diversity. As an outgrowth of his professional and personal experiences, Richard Bucher wrote this book to assist the reader in comprehending and respecting diversity and also to inform the reader of methods of developing diversity consciousness.

The author's central premise is that diversity is not an option because people and cultures are overlapping and becoming more interdependent. He wrote that "Human diversity, in its many shapes, forms and colors, is an integral part of our everyday life" (p. xi). And the societal consequence of a diverse and multi-cultural environment, there is a real, concrete need that " ...each of us desperately needs to develop our diversity consciousness: our awareness, understanding, and skills in the area of diversity" (p. xi). This is his attempt to prepare the reader to appreciate this increasingly large, diverse social world.

Throughout the book, Bucher uses several instructional tools that are beneficial in further enhancing topics and issues related to diversity. He uses student perspectives throughout the book in the form of direct quotes from students he has had in classes throughout his career. These quotes are always in-line with the topic he has discussed in the previous section. Secondly, he provides "journal entry" questions, which require the reader to think through various aspects of diversity. These questions require the reader to utilize self-reflection and evaluation to guide their thoughts regarding their diversity consciousness. Finally, Bucher uses a variety of exercises at the end of the chapter as a tool. Due to the format of this method, instructors can effectively use the exercises as an instructional tool through which students can reflect on issues both individually, within groups, as well as through technology.

Chapter 1- "Diversity: An Overview", provides a definition of diversity that the author

Myron L. Pope, Ed.D. is an Assistant Professor of Adult and Higher Education at the University of Oklahoma in Norman, OK. 
states is "all of the ways in which people are different" (p. 2). This definition is further explained in relation to culture. The author emphasizes how the cultural landscape has changed over the past two hundred years in the United States and how its citizens have reacted to it. Globalization and technology have further expanded this cultural landscape, and society is confronted with more distinguishing dimensions of diversity. The author closes the chapter by defining some of those dimensions, negating some long standing myths regarding diversity, and explaining diversity education. This chapter is well designed to assist the reader in developing a new diversity consciousness.

Chapter 2: "Diversity Skills and Success", examines the many definitions of success based on individual socio-cultural orientations. The chapter also focuses on the three components necessary in building diversity consciousness as defined by Buchner: awareness, understanding, and diversity skills. He emphasizes that through the acquisition and utilization of diversity skills, individuals will be better able to think through and value human differences in any setting. This will ultimately improve success in all facets of human life, including, but not limited to, school and work. The chapter concludes by outlining the benefits of using diversity skills with to various constituents, and consequently, the detriment of inadequate diversity skills.

Chapter 3: "Personal and Social Barriers to Success", focuses on individual and societal dimensions that inhibit the success of accepting diversity. Barriers such as stereotypes, ethnocentrism, limited perceptions, prejudice, prejudice plus power, and discrimination are defined and explored as they relate to the success of individuals and society. Some strategies and suggestions are provided as teaching tools to assist the reader in overcoming diversity barriers. The chapter concludes by emphasizing that it is important to acknowledge and overcome these diversity barriers.

Chapter 4: "Developing Diversity Consciousness", focuses on getting the reader to understand that diversity consciousness is not tolerating human differences, but rather attempting to understand, respect, and value differences. Once this effort is achieved, individuals will behave and think differently in a more accommodating manner. Even though individuals initially begin to change habits and attitudes, it is paramount that continued adaptation and respect for human differences continue, as diversity consciousness is dynamic and ever changing.

Chapter 5: "Communicating in a Diverse World", explores the relationship between communication and diversity consciousness. As society becomes more diversity conscious, modes of communication will become less channel specific, more open to all, and in a sense, more perfected due to an awareness and exhibition of skills that acknowledge human differences. This increased sensitivity will ultimately provide unlimited possibilities to communicate in any setting.

Chapter 6: "Teamwork", defines teams and analyzes different types of teams and factors 
that are both instrumental and detrimental to the success of teams. The author explores the dynamics of team leadership, especially in efforts to enhance diversity consciousness and the strategies necessary to be an effective leader. Since conflict can tear a team apart, approaches to conflict management are discussed within the chapter.

Buchner does an excellent job in relaying the significance of diversity consciousness in building community by accepting and respecting human differences. Even though accepting human difference is a decision left to the individual, it is important for leaders to promote diversity in schools and at work. This is especially the case in the area of orientation services. As new students transition into a new environment, they must feel confident or their chances of matriculation, retention, graduation, and success will be decreased. Through the training of orientation group leaders and members based on the strategies outlined by Buchner, orientation programs will be successful in welcoming all new students as they transition into their new environments.

This book would also be beneficial in training new students on diversity in an orientation class. Colleges and universities have long played a role in correcting many social ills, and through diversity training, students will gain or understanding of and appreciation for diversity. 